Métodos de Preparação Industrial de Solventes e Reagentes Químicos

\title{
Hexafluoroisopropanol (HFIP, CAS No 920-66-1)
}

Barros, J. C.*; Gonçalves, R. S. B.; Lima, C. H. S.; Monte, P. M.; Silva, T. L.; Souza, A. L. F.

Rev. Virtual Quim., 2020, 12 (5), 1334-1341. Data de publicação na Web: 19 de Agosto de 2020

http://rvq.sbq.org.br

\section{Hexafluoroisopropanol (HFIP, CAS No 920-66-1)}

Abstract: Hexafluoroisopropanol is a fluorinated compound quite unknown in Brazil which finds applications as solvent and chemical intermediate in drug production. This work shows its discovery, main properties, applications and finally several methods of production at industrial scale.

Keywords: Hexafluoroisopropanol; HFIP; fluorinated solvent; organofluorine; fluoroketones; hexafluoroacetone

\section{Resumo}

Hexafluoroisopropanol é um composto fluorado pouco explorado no Brasil que possui aplicações como solvente e intermediário de fármacos. Este artigo apresenta sua descoberta, propriedades, aplicações e finalmente diferentes métodos de preparação de HFIP em escala industrial.

Palavras-chave: Hexafluoroisopropanol; HFIP; solvente fluorado; organofluorado; fluorocetonas; hexafluoroacetona

* Universidade Federal do Rio de Janeiro, Centro de Tecnologia, Instituto de Química, Bloco A, 70 andar, Avenida Athos da Silveira Ramos 149, CEP 21941-909, Cidade Universitária, Rio de Janeiro-RJ, Brasil.

Mibarros@iq.ufri.br

DOI: $10.21577 / 1984-6835.20200104$ 


\section{Hexafluoroisopropanol (HFIP, CAS No 920-66-1)}

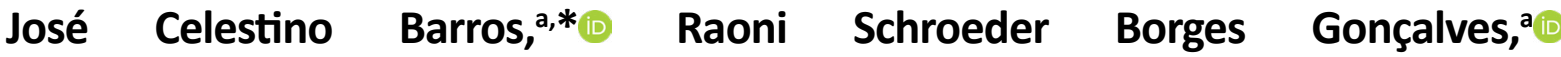 Camilo Henrique da Silva Lima, ${ }^{a}$ Pamella Moreira Monte, ${ }^{a}$ Tiago Lima da Silva, Andréa Luzia Ferreira de Souza ${ }^{a}$}

a Universidade Federal do Rio de Janeiro, Centro de Tecnologia, Instituto de Química, Bloco A, 7o andar, Avenida Athos da Silveira Ramos 149, CEP 21941-909, Cidade Universitária, Rio de Janeiro-RJ, Brasil.

b Universidade Federal do Rio de Janeiro, Campus Macaé, Avenida Aluizio da Silva Gomes 50, Granja dos Cavaleiros, CEP 27930-560, Macaé-RJ, Brasil.

*ibarros@iq.ufri.br

Recebido em 14 de Abril de 2020. Aceito para publicação em 3 de Agosto de 2020.

\section{Introdução}

\section{Propriedades}

\section{Aplicações}

\section{Métodos de Preparação}

\section{Conclusão}

\section{Introdução}

Hexafluoroisopopanol - HFIP (1) (Figura 1), cujo nome oficial é 1,1,1,3,3,3-hexafluoro-2-propanol, é um fluoroálcool de massa molar 168,05 g mol-1 $^{-1}$ pouco explorado em Química Orgânica no Brasil. Uma busca sistemática em março de 2020 nas bases Scifinder e Web of Science por "HFIP OR hexafluoroisopropanol OR hexafluoroisopropanol OR hexafluoro-2-propanol" mostrou apenas 26 artigos e 10 patentes de pesquisadores no Brasil que relacionam seu uso. ${ }^{1}$
Embora houvesse relatos anteriores de preparação do HFIP, sua popularização nos países do ocidente se deu em meados dos anos 60 e pode ser considerada um acaso (serendipidade). Enquanto tentavam preparar fluorotiocetonas, Middleton e colaboradores na empresa DuPont reduziam fluorocetonas a fluoroálcoois com $\mathrm{LiAlH}_{4}$. Porém, não era possível separar os fluoroálcoois obtidos do solvente da reação (THF) por destilação ou cristalização graças a formação de complexos estáveis com espécies doadoras de ligação de hidrogênio. ${ }^{2}$

Assim, o grupo sugeriu empregar estes fluoroálcoois na superfície de polímeros para torná-<smiles>OC(C(F)(F)F)C(F)(F)F</smiles>

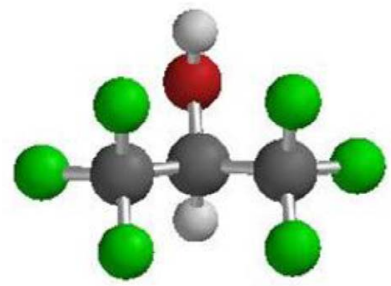

Hexafluoroisopropanol

HFIP 1

Figura 1. Estrutura química do hexafluoroisopropanol - HFIP (1) e representação tridimensional 
los hidrofóbicos. Nada poderia ser mais inverídico, já que segundo o próprio inventor, os polímeros testados "se dissolviam como açúcar em água" nos fluoroálcoois. ${ }^{2}$

Percebendo a oportunidade de mercado, a empresa DuPont comercializou um destes novos fluoroálcoois - hexafluoroisopropanol (HFIP) inicialmente como um solvente para soldagem de polímeros como poliamidas, poliésteres e poliéteres, bem como solvente para controle analítico dos polímeros. Além disso, fluoroálcoois deuterados foram empregados como solventes para estudos de RMN de polímeros. ${ }^{3}$

\section{Propriedades}

HFIP é um líquido incolor e um solvente polar $\left(E_{T}^{\mathrm{N}}=1,068\right)$ miscível em água. Entre 360 solventes testados, o HFIP foi o único a ultrapassar a escala Parâmetro de Polaridade de Solvente de Reichert $\left(\mathrm{E}_{\mathrm{T}}^{\mathrm{N}}\right)$ que vai de zero $\left(\mathrm{Me}_{4} \mathrm{Si}\right)$ a $1,0\left(\mathrm{H}_{2} \mathrm{O}\right)$. Outra característica é a acidez do hidrogênio de sua hidroxila $(p K a=9,3)$ quando comparado ao seu álcool análogo, o álcool isopropílico ( $\mathrm{pKa}=16,5$ ). HFIP tem alto poder de doação de ligação de hidrogênio $(\alpha=1,96)$, alto poder ionizante $(Y=3,82$ na escala de Grunwald-Winstein) e seu poder de solvatação através de ligações de hidrogênio é tão alto que ele forma complexos com THF ou outros solventes básicos. Outra característica de HFIP é não ser nucleofílico ( $\mathrm{N}=-4,23$ na escala de GrunwaldWinstein), nem aceptor de ligação de hidrogênio $(\beta=0)$. A Tabela 1 llustra estas e outras propriedades de HFIP. ${ }^{4} \mathrm{O}$ HFIP é ainda considerado um material corrosivo, que causa severas queimaduras na pele e nos olhos e possui toxicidade reprodutiva. ${ }^{5}$

$O$ preço deste reagente no Brasil em escala de laboratório (Aldrich) é de aproximadamente
$\mathrm{R} \$ \mathbf{4} 4.500,00 / \mathrm{Kg}^{5}$ e os maiores produtores mundiais incluem Chemours, Halocarbon, Gujarat Fluorochemicals Limited, Sanming HexaFluo Chemicals, Shanghai Nuohey Chemical Technology e Lianyungang Tetrafluor New Materials. ${ }^{6}$

\section{Aplicações}

HFIP possui diversas aplicações como solvente nas reações de epoxidação, ${ }^{7}$ oxidação, ${ }^{8}$ hidrogenação de aromáticos, ${ }^{9}$ ciclização, ${ }^{10}$ e mais recentemente na ativação de ligações $\mathrm{C}-\mathrm{H} \cdot{ }^{11}$ Outras aplicações incluem estabilização de cátions radicais, ${ }^{12}$ polimerização radicalar, ${ }^{13}$ análise de polímeros por MALDI-TOF ou $\mathrm{GPC} / \mathrm{SEC}^{14}$ e estabilização da conformação de peptídeos e proteínas, ${ }^{15}$ enquanto diversas outras aplicações foram revisadas na literatura recentemente. ${ }^{4,16}$

Comointermediário de síntese, o principal uso de HFIP é na produção do sevoflurano (2), um Insumo Farmacêutico Ativo (IFA). Este IFA é um anestésico inalatório para indução e manutenção de anestesia geral que possui diversas características desejáveis: não inflamável, de rápida indução e recuperação do paciente, mínima irritação do sistema respiratório e rápida eliminação. ${ }^{17}$

A produção industrial deste anestésico desenvolvida pela empresa Baxter envolve a reação de HFIP (1) com paraformaldeido e HF em presença de $\mathrm{H}_{2} \mathrm{SO}_{4}$ (Esquema 1). ${ }^{18}$ Porém, esta reação gera diversos subprodutos e requer extensivos processos de purificação. ${ }^{19}$ Assim, a empresa farmoquímica brasileira Cristália desenvolveu uma rota em duas etapas baseada na reação de HFIP com paraformaldeído, cloreto de tionila e ácido sulfúrico para obtenção de sevoclorano, que em seguida é fluorado empregando fluoreto de potássio e iodeto de potássio para obter sevofluorano. ${ }^{20}$ (Esquema 2).

Tabela 1. Propriedades de HFIP ${ }^{4}$

\begin{tabular}{lccc}
\hline Parâmetro & Valor & Parâmetro & Valor \\
\hline $\mathrm{PE}\left({ }^{\circ} \mathrm{C}\right)$ & 58,6 & a Polaridade $\left(\mathrm{E}_{\mathrm{T}}^{\mathrm{N}}\right)$ & 1,068 \\
$\mathrm{PF}\left({ }^{\circ} \mathrm{C}\right)$ & -5 & ${ }^{\mathrm{b}}$ Poder ionizante $(\mathrm{Y})$ & 3,82 \\
Densidade $(\mathrm{d})$ & 1,605 & ${ }^{\mathrm{c}}$ Doador de ligação de hidrogênio $(\alpha)$ & 1,96 \\
pKa & 9,3 & ${ }^{c}$ Acceptor de ligação de hidrogênio $(\beta)$ & 0 \\
${ }^{\mathrm{b}}$ Nucleofilicidade $(\mathrm{N})$ & $-4,23$ & Constante de autoassociação $\left(\mathrm{dm}^{3} \mathrm{~mol}^{-1}\right)$ & 0.13 \\
Constante dielétrica $(\varepsilon)$ & 16,7 & & \\
\hline
\end{tabular}

${ }^{a}$ Escala Reichert de polaridade . ${ }^{b}$ Escala de Grunwald-Winstein. ${ }^{c}$ Escala Kamlet-Taft 
<smiles>OC(C(F)(F)F)C(F)(F)F</smiles>

Hexafluoroisopropanol HFIP 1

$$
\underset{\mathrm{H}_{2} \mathrm{SO}_{4}, 65^{\circ} \mathrm{C}}{\stackrel{\left(\mathrm{CH}_{2} \mathrm{O}\right)_{\mathrm{n}}, \mathrm{HF}(\mathrm{g})}{\longrightarrow}}
$$<smiles>FCOC(C(F)(F)F)C(F)(F)F</smiles>

Sevoflurano 2 $71 \%$

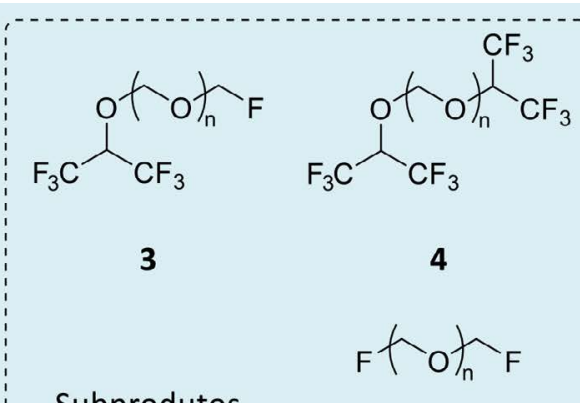

Subprodutos

5
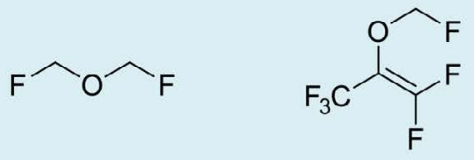

7

Esquema 1. Rota de produção industrial de sevoflurano $2^{18}$

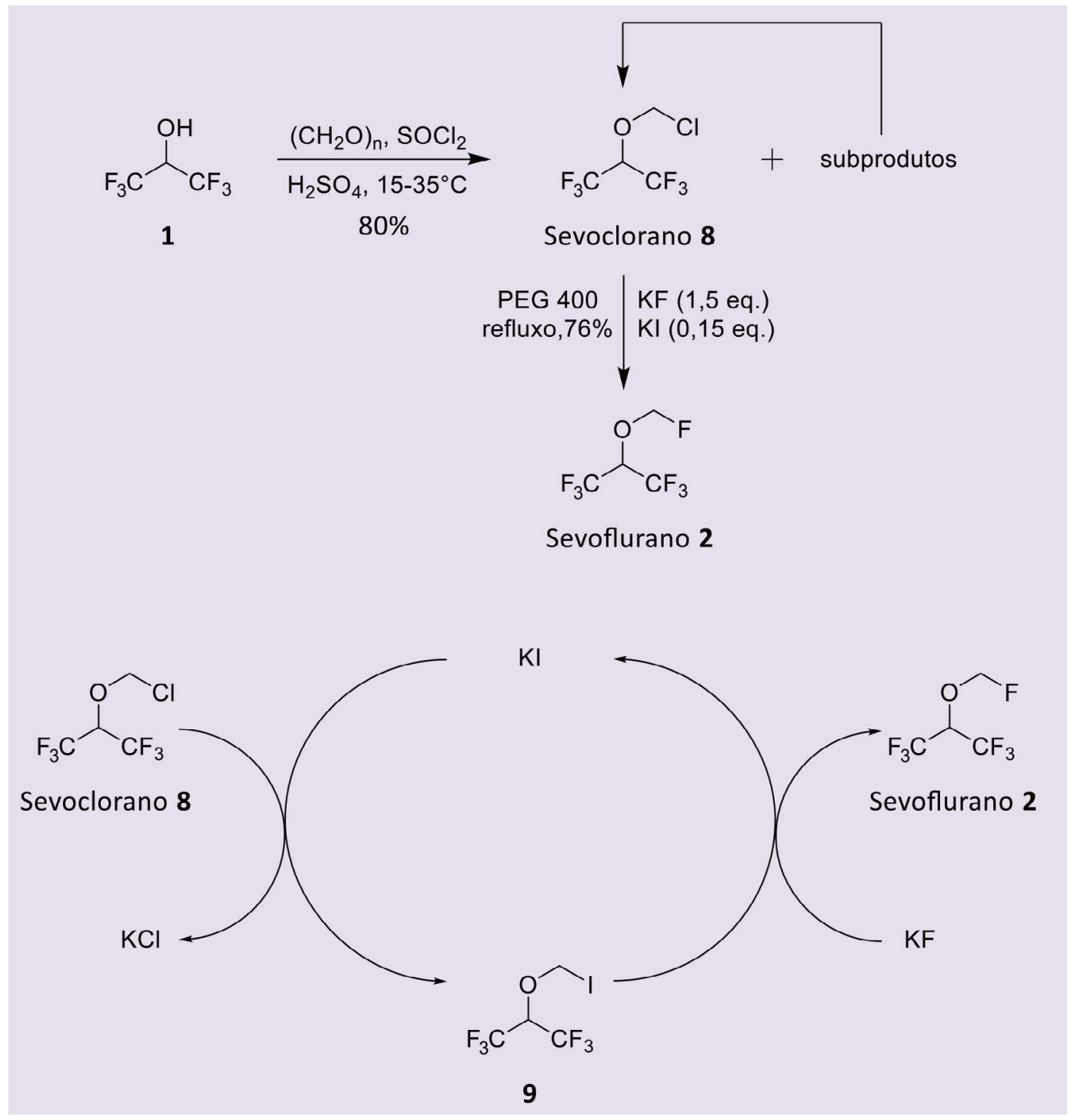

Esquema 2. Rota de produção industrial do sevoflurano (2) desenvolvida no Brasil ${ }^{20}$ 


\section{Métodos de Preparação}

Existem diversos métodos para preparação de HFIP (1), como a reação de substituição nucleofílica entre cloro-1,1,1,3,3,3,-hexafluoropropano (10) e acetato de potássio seguida por remoção do grupo acetila $^{21}$ ou a descarboxilação do hidroxiéster (11) empregando trietilamina em etilenoglicol. ${ }^{22}$ Porém, a reação mais estudada é a redução da hexafluoroacetona (HFA) (12) ou de seu hidrato $(13),{ }^{2,23-28}$ e as mais utilizadas em escala industrial empregam hidrogênio molecular em presença de catalisadores de metais de transição (Esquema 3).

Embora possa parecer simples reduzir hexafluoroacetona (12) para hexafluoroisopropanol (1) empregando hidrogênio molecular, na prática, impurezas $(14,16-17)$ no reagente de partida e subprodutos $(\mathbf{1 5}, \mathbf{1 7}-19)$ da reação podem dificultar este processo e diversas reações laterais foram relatadas na literatura (Esquema 4). ${ }^{29}$

Katsuhara e colaboradores $^{30}$ realizaram hidrogenação do hidrato de HFA (13) na fase líquida em reatores batelada de até $100 \mathrm{La} 70^{\circ} \mathrm{C}$ empregando como catalisadores $0,5 \% \mathrm{~m} / \mathrm{m}$ de Pd/C ou Ni-Raney ${ }^{\circledR}$. Os rendimentos obtidos foram de $99 \%$ para o processo empregando paládio e $69 \%$ para níquel. Neste processo hidróxidos de sódio e de alumínio foram adicionados em quantidades catalíticas $(0,13 \%$ e $0,5 \% \mathrm{~m} / \mathrm{m}$ ) para suprimir a formação de fluoreto que pudesse danificar tubulações de vidro.

De maneira similar, Nakamichi e colaboradores empregaram concomitantemente $\mathrm{Pd} / \mathrm{Al}_{2} \mathrm{O}_{3}$ e $\mathrm{Ru}$ /
$\mathrm{Al}_{2} \mathrm{O}_{3}(0.12 \%$ e $5 \% \mathrm{~m} / \mathrm{m})$, hidróxido de alumínio e bicarbonato de sódio em reatores de $5 \mathrm{~L}$ para hidrogenação de hidrato de HFA a $95{ }^{\circ} \mathrm{C}$, obtendo assim um rendimento de $95 \%$ de HFIP. ${ }^{31}$

Tradicionalmente, processos em batelada possuem capacidade limitada de produção, tempos longos de reação, e apresentam problemas como atrito do catalisador nas paredes do reator, dificuldade de remoção e recuperação de finas partículas do catalisador. Além disso, processos em batelada requerem utilização de grandes quantidades dos reagentes de partida no reator levando a preocupações com a segurança do processo.

Visando resolver estas questões, Tieri e colaboradores desenvolveram um processo em fase líquida contínuo. ${ }^{29,32}$ Este processo teve como inovação o uso de dois reatores, o primeiro um autoclave com agitação de $1 \mathrm{~L}$ para preparação de uma corrente líquida composta por $\mathrm{H}_{2}$, HFA (12) e HFIP, e o segundo um reator tubular em aço de leito fixo contendo catalisador $\mathrm{Ni} / \mathrm{Al}_{2} \mathrm{O}_{3}$. De maneira resumida, o fluxograma deste processo conta ainda com tubulações para transporte de matérias primas e produtos, bombas, válvula, trocadores de calor e um separador líquido-vapor para obtenção do produto (Figura 2).

O uso do reator de fluxo continuo com excesso de hidrogênio dissolvido na corrente de entrada e recirculação dessa corrente faz com que este processo ocorra em condições praticamente isotérmicas, com reduzida formação de subprodutos ou coque, aumentando a vida útil

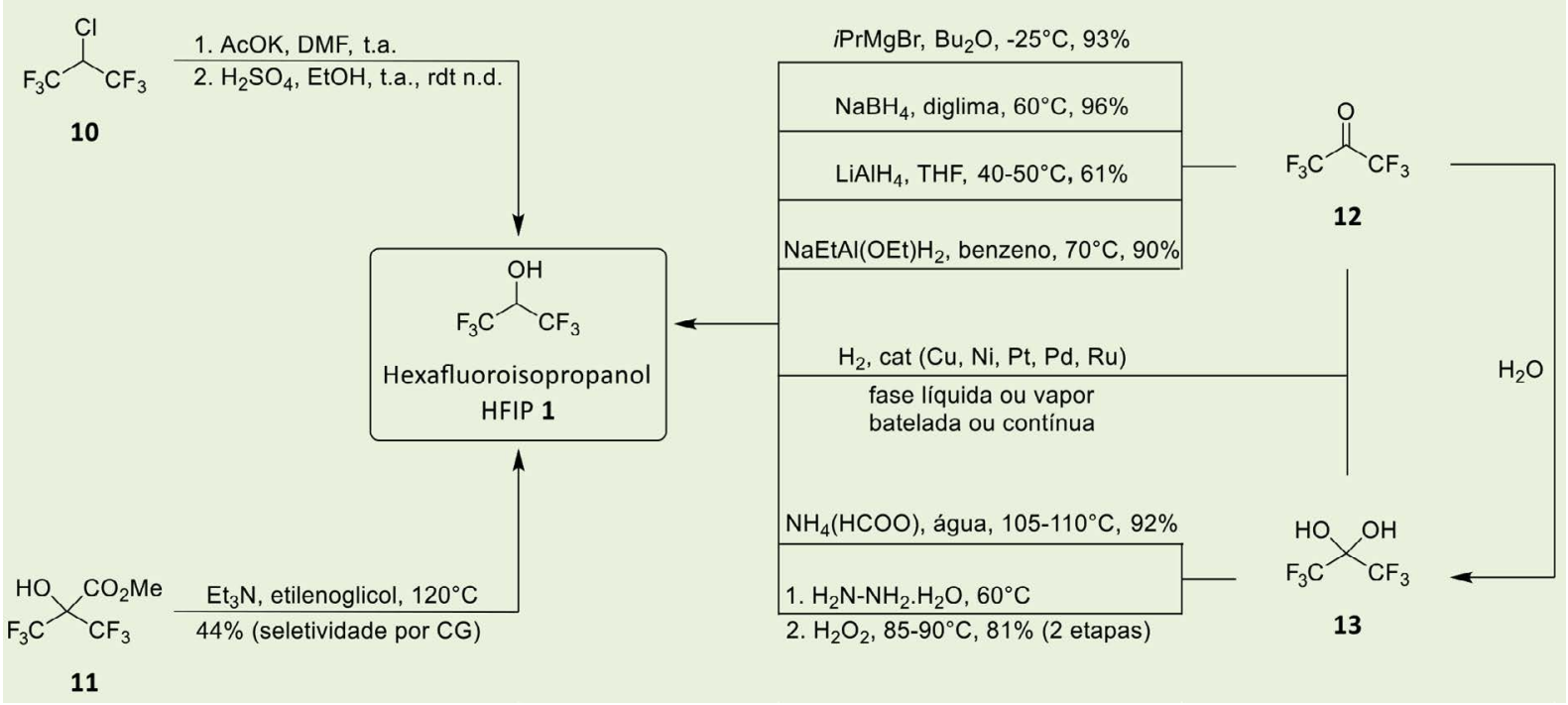

Esquema 3. Métodos de preparação de HFIP (1) através de reações envolvendo os compostos 10-13 


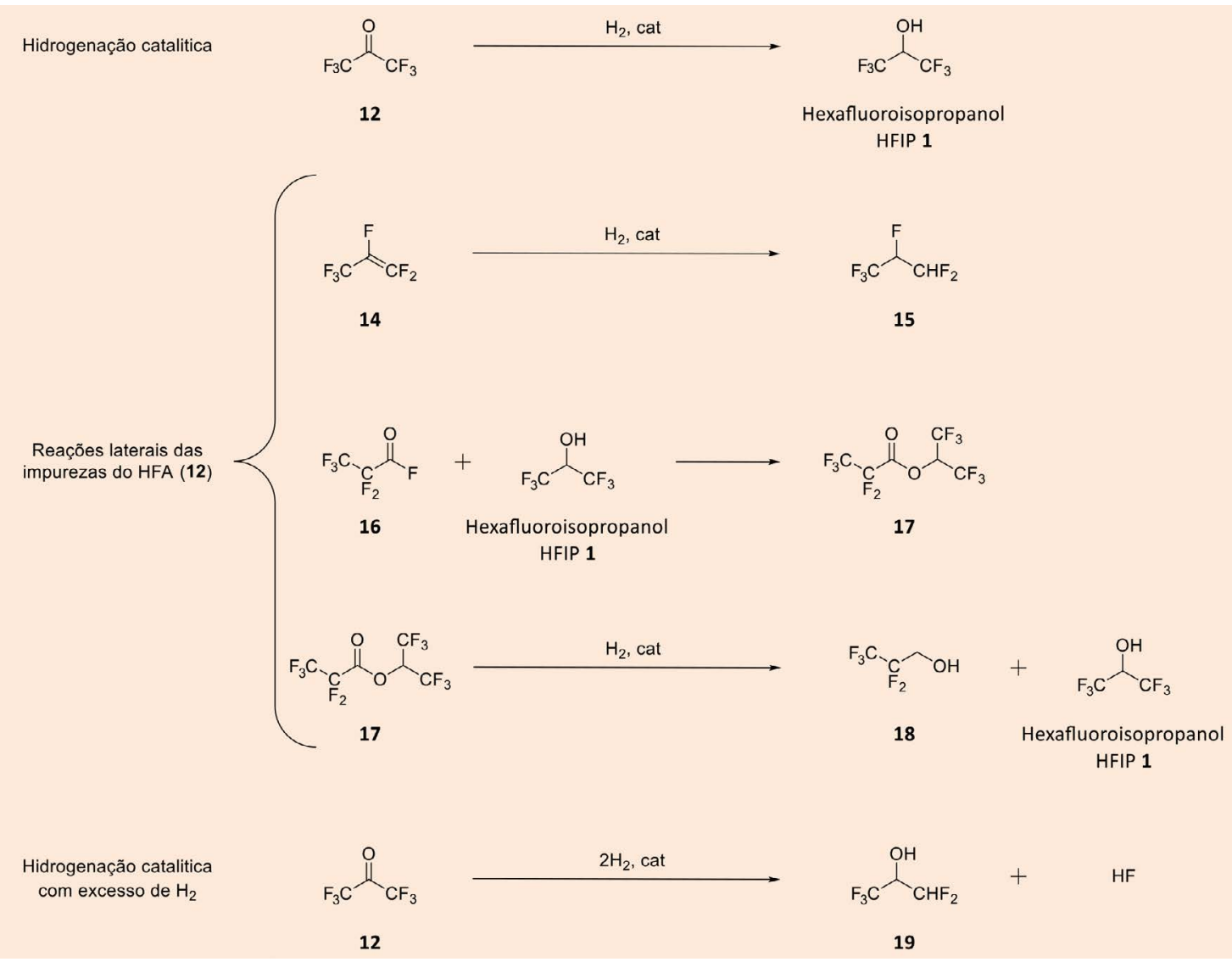

Esquema 4. Redução de HFA a HFIP e possíveis reações laterais ${ }^{29}$

do catalisador e finalmente evitando grande quantidade de HFA no reator, o que garante maior segurança ao processo.

Ensaios preliminares em batelada indicaram como melhor catalisador o $\mathrm{Ni} / \mathrm{Al}_{2} \mathrm{O}_{3}(2,5 \% \mathrm{~m} / \mathrm{m})$ e rendimento de $96 \%$. No sistema contínuo e com alimentação de $150 \mathrm{~g} / \mathrm{h}$ de HFA, os melhores resultados foram obtidos a $120{ }^{\circ} \mathrm{C}$ e pressão de
600 psi com fluxo ascendente no reator (up-flow), fornecendo conversão $>99,5 \%$ para o HFIP. A reciclabilidade do catalisador foi excelente, pois se apresentou ativo após $353 \mathrm{lb} \mathrm{HFA} / \mathrm{lb}$ catalisador.

Finalmente, os processos contínuos em fase vapor são os mais empregados na literatura. Eles podem fazer uso de metais como cobre, níquel, platina ou paládio, nas mais diversas condições (Tabela 2).

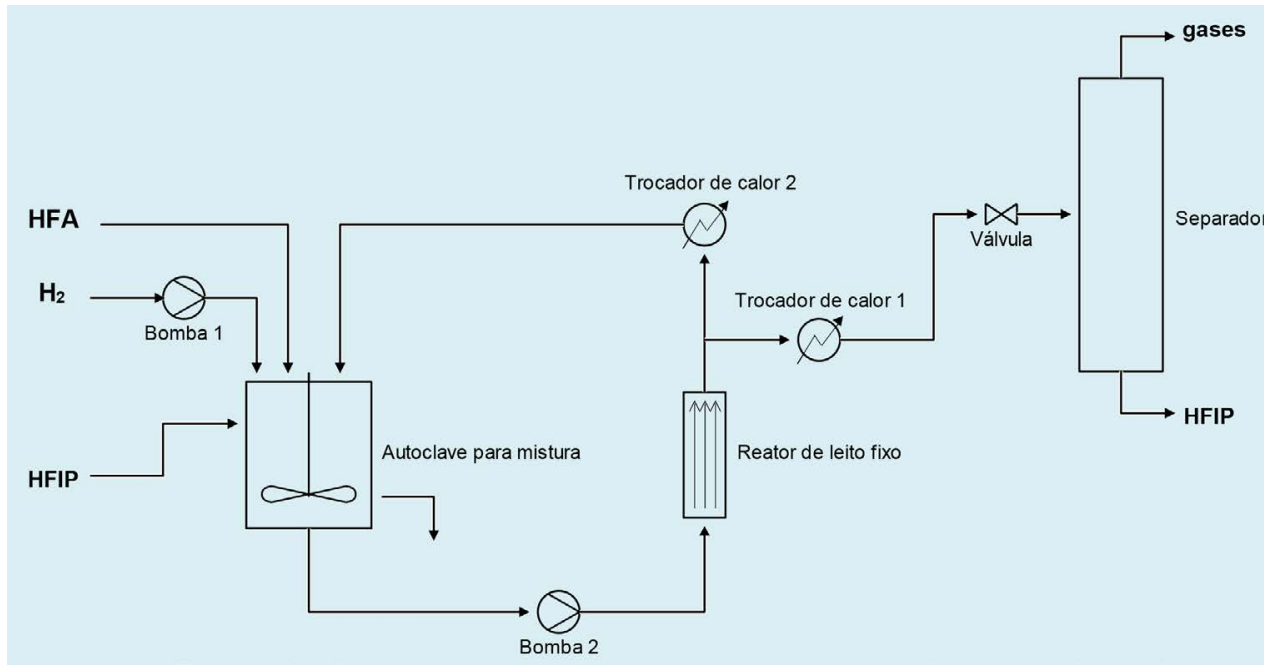

Figura 2. Fluxograma de processo contínuo em fase líquida para preparação de HFIP (Fonte: adaptada de ${ }^{32}$ ) 
Tabela 2. Preparação de HFIP por hidrogenação em fase vapor

\begin{tabular}{lccccccc}
\hline Reação & Catalisador & $\mathbf{T}\left({ }^{\circ} \mathbf{C}\right)$ & Vazão $(\mathbf{g} / \mathbf{h})$ & $\begin{array}{c}\text { Material } \\
\text { do reator }\end{array}$ & $\begin{array}{c}\text { Dimensões do reator } \\
(\mathbf{I} \times \mathbf{~ d . i .})(\mathbf{c m} \mathbf{~ c m})\end{array}$ & Rdt (\%) & Referência \\
\hline 1 & $\mathrm{Cu} / \mathrm{Cr}_{2} \mathrm{O}_{3}$ & 300 & $104 \mathrm{~g} / \mathrm{h}$ & Níquel & $96 \times 2,5$ & 40 & 33 \\
2 & $\mathrm{Ni} / \mathrm{C}$ & 40 & $40 \mathrm{~g} / \mathrm{h}$ & Pyrex & n.d. $\times 2,5$ & $>99$ & 34 \\
3 & $\mathrm{PtO}_{2}$ & $110-150$ & $17 \mathrm{~g} / \mathrm{h}$ & Pyrex & n.d. & 90 & 24 \\
4 & $\mathrm{Pd} / \mathrm{C}$ & 200 & $168 \mathrm{~g} / \mathrm{h}$ & Pyrex & $45 \times 1,9$ & 75 & 35 \\
5 & $\mathrm{Pd} / \mathrm{Al}_{2} \mathrm{O}_{3}$ & $150-157$ & $19 \mathrm{~g} / \mathrm{h}$ & Pyrex & $33 \times 1,9$ & $>99$ & 36 \\
\hline
\end{tabular}

n.d. não descrito

Emboraos processosemfasevaporrepresentem avanços em relação aos processos em batelada, como maior produtividade e segurança, pois não requerem grandes quantidades de reagente dentro do reator, eles também apresentam problemas, como por exemplo um gradiente de temperatura no leito fixo do catalisador causado pela reação exotérmica que pode gerar pontos quentes (hot spots) e portanto subprodutos como o HF e desativação dos catalisadores. ${ }^{29}$

\section{Conclusão}

Pode-seconcluirentãoque oHFIP, como solvente ou como intermediário de reação se encontra pouco explorado no Brasil apesar de possuir diversos métodos de produção, destacando-se as reações de hidrogenação catalítica em fase vapor, porém novos processos em fase líquida de maneira contínua surgiram para aliar alta produtividade e segurança a eliminação dos problemas observados na produção em fase vapor.

\section{Referências Bibliográficas}

${ }^{1}$ a) Clarivate Analytics. Web of Science Core Collection, Philadelphia, 2020. b) Chemical Abstract Service. Scifinder, Columbus, 2020.

${ }^{2}$ Middleton, W. J. Em Fluorine Chemistry at the Millennium. Fascinated by Fluorine; Banks, R. E., ed.; Elsevier: Amsterdam, 2000, cap. 21. [CrossRef]

${ }^{3}$ a) Middleton, W. J. US 3,418,3371964 (CA 60:7000). b) Middleton, W. J. US 3,245,944 1966 (CA 64:93973).

${ }^{4}$ Bégué, J.-P.; Bonnet-Delpon, D.; Crousse, B. Fluorinated alcohols: a new medium for selective and clean reaction. Synlett 2004, 18. [CrossRef]

${ }^{5}$ Sítio da Aldrich (Merck). Disponível em <https:// www.sigmaaldrich.com/brazil.html>. Acesso em 31 Março 2020.
${ }^{6}$ Sítio da empresa Market Watch. Disponível em: <https://www.marketwatch.com/press-release/ hexafluoroisopropanol-hfip-market-2019-withtop-countries-data-market-size-industry-trendsgrowth-insight-share-competitive-analysisstatistics-regional-and-global-industry-forecastto-2025-2019-10-02>. Acesso em: 31 Março 2020. ${ }^{7}$ a) van Vliet, M. C. A.; Arends, I. W. C. E.; Sheldon, R. A. Fluorinated alcohols: effective solvents for uncatalysed epoxidations with aqueous hydrogen peroxide. Synlett 2001, 2001, 0248. [CrossRef] b) Berkessel, A.; Adrio, J. A. Dramatic acceleration of olefin epoxidation in fluorinated alcohols: activation of hydrogen peroxide by multiple $\mathrm{H}$-bond networks. Journal of the American Chemical Society 2006, 128, 13412. [CrossRef] [PubMed]

${ }^{8}$ Berkessel, A. Em Modern Oxidation Methods, 2nd ed., Bäckvall, J.-E., ed.; Wiley-VCH, Weinheim, 2010, Cap. 4. [CrossRef]

9 Fache, F.; Piva, O. Hexafluoroisopropanol: a powerful solvent for the hydrogenation of functionalized aromatic compounds. Synlett 2004, 1294. [CrossRef]

${ }^{10}$ a) Saito, A.; Kanno, A.; Hanzawa, Y. Synthesis of 2,3-disubstituted indoles by a rhodiumcatalyzed aromatic amino-Claisen rearrangement of $\mathrm{N}$-propargyl anilines. Angewandte Chemie International Edition 2007, 46, 3931. [CrossRef] [PubMed] b) Yokota, M.; Fujita, D.; Ichikawa, J. Activation of 1,1-difluoro-1-alkenes with a transitionmetal complex: palladium(II)-catalyzed FriedelCrafts-type cyclization of 4,4-(difluorohomoallyl) arenes. Organic Letters 2007, 9, 4639. [CrossRef] [PubMed]

${ }^{11}$ Sinha, S. K.; Bhattacharya, T.; Maiti, D. Role of hexafluoroisopropanol in $\mathrm{C}-\mathrm{H}$ activation. Reaction Chemistry \& Engineering 2019, 4, 244. [CrossRef] b) D'Amato, E. M.; Börgel, J.; Ritter, T. Aromatic C-H amination in hexafluoroisopropanol. Chemical Science 2019, 10, 2424. [CrossRef] [PubMed] 
12 Eberson, L.; Hartshorn, M. P.; Persson, O.; Radner, F. Making radical cations live longer. Chemical Communications 1996, 2105. [CrossRef] ${ }^{13}$ a) Yamada, K.; Nakano, T.; Okamoto, Y. Free-radical polymerization of vinyl esters using fluoroalcohols as solvents: Effect of monomer structure on stereochemistry. Journal of Polymer Science, Part A: Polymer Chemistry 1999, 37, 2677. [CrossRef] b) Wan, D.; Satoh, K.; Kamigaito, M.; Okamoto, Y. Xanthatemediated radical polymerization of $N$-vinylpyrrolidone in fluoroalcohols for simultaneous control of molecular weight and tacticity. Macromolecules 2005, 38, 10397. [CrossRef]

${ }^{14}$ Hoteling, A. J.; Mourey, T. H.; Owens, K. G. Importance of solubility in the sample preparation of poly(ethylene terephthalate) for MALDI TOFMS. Analytical Chemistry 2005, 77, 750. [CrossRef] [PubMed] b) Nguyen, T. Q. Polyamides: GPC/SEC analysis. Em Encyclopedia of Chromatography, 3rd ed., Cazes, J., ed.; CRC Press: Boca Raton, 2010, 1846. [CrossRef] c) Ferrito, S. J. Polyesters: GPC/SEC analysis. Em Em Encyclopedia of Chromatography, 3rd ed., Cazes, J., ed.; CRC Press: Boca Raton, 2010, 1853. [CrossRef]

${ }^{15}$ a) Hirota, N.; Goto, Y.; Mizuno, K. Cooperative $\alpha$-helix formation of $\beta$-lactoglobulin and melittin induced by hexafluoroisopropanol. Protein Science 1997, 6, 416. [CrossRef] [PubMed] b) Hong, D.-P.; Hoshino, M.; Kuboi, R.; Goto, Y. Clustering of fluorine-substituted alcohols as a factor responsible for their marked effects on proteins and peptides. Journal of the American Chemical Society 1999, 121, 8427. [CrossRef].

${ }^{16}$ a) Colomer, I.; Chamberlain, A. E. R.; Haughey, M. B.; Donohoe, T. J. Hexafluoroisopropanol as a highly versatile solvent. Nature Reviews Chemistry 2017, 1, 0088. [CrossRef] b) Khaksar, S. Fluorinated alcohols: a magic medium for the synthesis of heterocyclic compounds. Journal of Fluorine Chemistry 2015, 172, 51. [CrossRef]. c) An, X.-D.; Xiao, J. Fluorinated alcohols: magic reaction medium and promoters for organic synthesis. The Chemical Record 2020, 20, 142. [CrossRef] [PubMed] d) Phillips, A. J. 1,1,1,3,3,3-Hexafluoro-2-propanol, Em e-EROS Encyclopedia of Reagents for Organic Synthesis, Wiley-VCH, Weinheim, 2010. [CrossRef].

${ }^{17}$ a) Longnecker, D. E.; Murphy, F. L. Introduction to Anesthesiology, 8th ed., W. B. Saunders: Philadephia, 1992. b) Reddy, V. P. Em Organofluorine Compounds in Biology and Medicine, Reddy, V. P., ed.; Elsevier: Amsterdam, 2000, cap. 21.
${ }^{18}$ Coon, C. L.; Simon, R. L. US 4,469,898 1982 (CA 94:208358).

${ }^{19}$ Ramakrishna, K.; Behme, C.; Schure, R. M.; Bieniarz, C. A safe and efficient process for the synthesis of the inhalation anesthetic sevoflurane. Organic Process Research \& Development 2000, 4, 581. [CrossRef].

${ }^{20}$ Pacheco, O.; Teixeira, A. C.; Lima, E. L.; Boeckelmann, M. A. WO 2008037040 2008. (CA 166:490175)

${ }^{21}$ Braun, M. J.; Kasubke, M. EP 2626341 2013. (CA 159:320795)

${ }^{22}$ Sugiyama, A.; Suzuki, A.; Tomita, M. WO 2008146746 2008. (CA 150:5377)

${ }^{23}$ a) Kötzsch, H.-J. Chemische Berichte 1966, 99, 1143. [CrossRef] b) Knunyants, I. L.; Krasuskaya, M. P. SU 138604 1960. (CA 56:45588)

${ }^{24}$ a) Middleton, W. J.; Lindsey, R. V. Hydrogen bonding in fluoro alcohols. Journal of the American Chemical Society 1964, 86, 4948. [CrossRef] b) Middleton, W. J. US 3,227,674 1966. (CA 64:51590) ${ }^{25}$ Málek, J. Reductions by Metal Alkoxyaluminum Hydrides. Organic Reactions 1985, 34. [CrossRef]

${ }^{26}$ Knunyants, I. L.; Gambaryan, N. P.; Ch'ingyun, C.; Rokhlin, E. M. Some reactions of hexafluoroacetone. Russian Chemical Bulletin 1962, 11, 633. [CrossRef]

${ }^{27}$ Igumnov, S. M.; Vyazkov, V. A. RU 23469242007 (CA 150:239797).

${ }^{28}$ Igumnov, S. M.; Sin'ko, A. V.; Don, V. L. $R U$ 25476542014 (CA 162:474837)

${ }^{29}$ Hagedorn, J. J.; Shankwitz, G. P.; Wolff, S. H.; Tieri, S. M.; Schultz, J. A.; Leib, T. M.; Sengupta, S. K. Topics in Catalysis 2014, 57, 1385. [CrossRef] ${ }^{30}$ Katsuhara, Y.; Nakamichi, T.; Kawai, T.; Nakazora, T. US 4,564,716 1986 (CA 103:214862)

${ }^{31}$ Morino, Yuzuru; Fujii, Shigeru; Nakamichi, Toshihiro; Akiba, Shinya; Takeda, Masaaki; Fujiwara, Masaki. EP 33237982016 (CA 166:238416)

${ }^{32}$ Tieri, S. M.; Hagedom, J. J.; Schultz, J. A.; Leib, T. M.; Wolff, S. H.; Sengupta, S. K.; Shankwitz, G. Paul. US 7,524,995 2009. (CA 150:474869)

${ }^{33}$ Hollander, J.; Woolf, C. BE 634368 1962. (CA 61:40081)

${ }^{34}$ Kawai, T.; Hibino, Y.; Maruyama, Y.; Negishi, A. GB 2073181 1981. (CA 96:122193)

${ }^{35}$ Regan, B. M. US 3, 702,872 1972.

${ }^{36}$ N.D. NL 6610936 1966. (CA 66:55024) 